\title{
Trabectedin and its potential in the treatment of soft tissue sarcoma
}

\author{
Philippe A Cassier' \\ Armelle Dufresne' \\ Jean-Yves Blay ${ }^{1,2,3}$ \\ Jérôme Fayette ${ }^{2,3}$ \\ 'Unité de Jour d'Oncologie Médicale \\ Multidisciplinaire, Hôpital Edouard \\ Herriot, Lyon, France; ${ }^{2}$ Département \\ d'Oncologie Médicale, Centre Léon \\ Bérard, Lyon, France; ${ }^{3}$ Unité INSERM \\ 590, Equipe Cytokine et Cancer, \\ Centre Léon Bérard, Lyon, France
}

\begin{abstract}
Trabectedin is a new marine-derived compound that binds the DNA minor groove and interacts with proteins of the DNA repair machinery. Phase I trials have established the standard regimen as $1500 \mu \mathrm{g} / \mathrm{m}^{2}$ 24-hour continuous infusion repeated every 3 weeks. Several phase II trials have shown response in 5\%-10\% of unselected patients with soft tissue sarcoma failing prior chemotherapy and disease stabilisation in $30 \%-40 \%$. Furthermore, prolonged disease control has been described in 15\%-20\% of patients. Toxicities are mainly haematological and hepatic with grade 3-4 neutropenia and thrombocytopenia observed in approximately $50 \%$ and $20 \%$ of patients respectively, and grade 3-4 elevation of liver enzymes observed in 35\%-50\% of patients treated with trabectedin. Current research focuses on the identification of predictive factors for patients with soft tissue sarcoma treated with trabectedin.
\end{abstract}

Keywords: chemotherapy, sarcoma, drug development, DNA repair

\section{Introduction}

Soft-tissue sarcomas (STS) are rare tumors of mesenchymal origin. Approximately $30 \%-50 \%$ of patients will experience metastatic relapse despite optimal locoregional treatment. In the setting of advanced or metastatic disease, systemic chemotherapy is indicated, although local therapies, such as surgery and radiation therapy, may achieve long term survival in selected patient (Clark et al 2005).

Doxorubicin alone or combined with ifosfamide has been the backbone of systemic chemotherapy for metastatic or advanced STS for more than 20 years with objective response observed in 15\%-35\% of patients (Wilson et al 1986; Antman et al 1993; Edmonson et al 1993). No standard treatment has yet emerged after failure of doxorubicin containing chemotherapy though some agents such as gemcitabine and gemcitabine-docetaxel combination have shown promising results (Patel et al 2001; Hensley et al 2002; Bay et al 2006). Trabectedin (ET743, YONDELIS ${ }^{\circledR}$, PharmaMar, Madrid, Spain), a tetrahydroisoquinoline alkaloid, is a natural product derived from the marine tunicate Ecteinascidia turbinata. ET743 has shown potent antitumor activity in preclinical studies both in vitro and in vivo on several solid tumors, including ovarian and breast cancer, melanoma, and sarcoma. These preclinical data have been confirmed in several phase II trials in breast and ovarian carcinoma, as well as STS. However, as with many other cytotoxic agents, the precise mechanism of trabectedin's cytotoxicity remains unclear.

\section{Mechanism of action}

Trabectedin (ET743) binds to the minor groove of DNA and alkylates guanine at the N2 position, whereas most alkylating agents bind guanine at position N7 or O6 in the major groove. Binding of trabectedin has been shown to be DNA-sequence 
specific, with GC rich triplets more frequently bound (David-Cordonnier et al 2005). Covalent binding of trabectedin induces DNA bending towards the major groove and a widening of the DNA minor groove (Takebayashi, Pourquier et al 2001). Modification of the DNA conformation leads to inhibition of activated transcription while constitutive transcription seems unaffected (Friedman et al 2002).

Several reports have underlined the importance of nucleotide-excision repair (NER) in the cytotoxicity of ET743, and more precisely the cell-killing ability of this drug has been linked to the transcription-coupled NER (TC-NER) (Takebayashi, Pourquier et al 2001; David-Cordonnier et al 2005; Herrero et al 2006). The DNA bending induced by the binding of trabectedin to the minor groove is detected by the TC-NER machinery, which in the repair process makes single strand breaks (SSBs) on each side of the lesion (David-Cordonnier et al 2005). These breaks are then made irreversible by the DNA-protein crosslinking capacities of trabectedin (Takebayashi, Goldwasser et al 2001). Recently Herrero et al (2006) suggested a slightly different model based on their observations made in the yeast model Schizosaccharomyces pompe. In this model, cells deficient for Rad13 (the yeast equivalent to the human XPG, which is an endonuclease of the NER system), were resistant to trabectedin, while those with an inactive Rad51 (a protein of the homologous recombination repair (HRR) pathway, involved in the repair of double stand breaks) were more sensitive to trabectedin than the wild-type cells. Based on these observations Herrero et al (2006) suggested the following sequence: trabectedin binds covalently to the DNA minor groove and the resulting adduct is recognised by the NER machinery, then the recruited Rad13 (XPG) protein binds to DNA and interacts with the minor groove-bound drug by means of an arginine residue located in the $\mathrm{COOH}$ terminus, other proteins of the NER machinery trying to repair the damage are then hijacked, forming larger, more toxic complexes. Lastly, during the $\mathrm{S}$ phase, the aforementioned complexes give rise to double stand DNA breaks, explaining the sensitivity of cells deficient for HRR pathway proteins (eg, Rad51) (Herrero et al 2006). In a recently published study, Soares et al (2007) showed that human cell lines deficient for the HRR proteins XRCC3, BRCA2, RAD51C and XRCC2 were 8 to 23 times more sensitive to treatment with trabectedin. Furthermore, their data shows that trabectedin-DNA adducts induce the formation of highly cytotoxic double strand breaks during the S-phase of the cell cycle (Soares et al 2007), thereby confirming, in human cell lines, the scenario suggested by Herrero et al (2006).

\section{Toxicity of trabectedin Common side effects of trabectedin in clinical trials}

Three phase I trials were conducted concomitantly in the United States, France and in Europe.

The American study, conducted by the teams of the Massachusetts General Hospital and the Dana-Farber Cancer Institute, tested the safety of trabectedin administered as a continuous infusion over 72 hours (Ryan et al 2001). In this trial enrolling 21 patients with various types of solid malignancies (including 6 sarcomas) the maximum tolerated dose (MDT) was $1200 \mu \mathrm{g} / \mathrm{m}^{2}$ over 72 hours every 3 weeks, the dose-limiting toxicity (DLT) was elevation of liver enzymes, which was also the most common toxicity, with grade 3 and 4 events observed in 41 and 14\% of cycles respectively. Grade 3-4 haematological toxicity was rare, occurring in only 2 patients. Fatigue was another frequently encountered side effect and was judged at least moderateto-severe in $24 \%$ of patients. Renal toxicity was observed in 4 patients, with 1 patient experiencing grade 3 and another experiencing grade 4 toxicity. No objective responses were seen in this trial.

In the French study trabectedin was administered as a 24 hours continuous infusion every 3 weeks (Taamma et al 2001). This trial enrolled 52 patients with various types of malignancies including 12 patients with sarcomas. Twenty seven patients were treated in an escalating dose phase and 25 more patients were treated in an extended phase at the recommended dose. The MDT was $1800 \mu \mathrm{g} / \mathrm{m}^{2}$ every 3 weeks, and the recommended dose was $1500 \mu \mathrm{g} / \mathrm{m}^{2}$. The DLT in this study was haematological (grade 4 neutropenia and thrombocytopenia), and was also the most common toxicity, grade 3-4 neutropenia and thrombocytopenia were seen in $52 \%$ and $28 \%$ of patients and $38 \%$ and $10 \%$ of cycles respectively at the recommended dose. Transient elevation of liver enzymes (AST/ALT) was the most common nonhaematological toxicity, grade 3-4 events were observed in $38 \%$ of patients. Asthenia was also a common side effect of treatment, but was severe (ie, grade 3-4) in only $8 \%$ of patients. Partial response occurred in 3 patients: one with breast cancer, one with osteosarcoma and one with STS.

In a third phase I trial conducted by the EORTC, the planned schedule for administration of trabectedin was a 1 hour infusion every 3 weeks, however the MDT was $1100 \mu \mathrm{g} / \mathrm{m}^{2}$ with this schedule and the study was extended 
by protocol amendment, allowing patients to be treated with a 3-hour schedule at higher doses of trabectedin, up to $1800 \mu \mathrm{g} / \mathrm{m}^{2}$ (Twelves et al 2003). Seventy-two patients were included in this study. The DLT were grade 4 neutropenia and thrombocytopenia and grade 3 fatigue using the 1-hour infusion and grade 4 thrombocytopenia, grade 3 fatigue and grade 3 bilirubinemia using the 3-hour infusion. The most common toxic side effects were haematological, with grade 3-4 neutropenia and thrombocytopenia in 20\% and $10 \%$ respectively. The most common non-haematological side effects were nausea and vomiting, fatigue and biochemical hepatic toxicity. Two objective tumor responses were observed, both in the 3-hour infusion cohort. Both of the responding patients had sarcoma (uterine leiomyosarcoma and stromal colon sarcoma).

Another phase I trial was reported a year later, using a daily bolus $\times 5$ days repeated every 3 weeks (VillalonaCalero et al 2002). Neutropenia and thrombocytopenia were the DLT and transient elevation of transaminases was also frequently observed. The maximum tolerated dose in this trial was $325 \mu \mathrm{g} / \mathrm{m}^{2} /$ day $\times 5$ every 3 weeks which is not significantly different from the recommended $1500 \mu \mathrm{g} / \mathrm{m}^{2} /$ cycle dose derived from other trials, indicating that tolerance of trabectedin was not enhanced by this schedule. Of note, antitumor activity was observed in a patient with STS (leiomyosarcoma).

Nausea and vomiting was a frequently encountered side effect in all trials, but was easily managed with $5 \mathrm{HT}_{3}-$ antagonists. The difference in the DLT and the MDT in the trial by Ryan et al and the other phase I trials reported by Taamma et al (2001) and Twelves et al (2003) was due to a slight difference in the definition of the DLT. Indeed in the American trial grade 3-4 transminitis was considered a
DLT if recovery was not complete by day 21 , while the 2 other trials allowed 28 days for complete recovery of grade 3-4 toxicity.

Based on the results of these different phase I trials the recommended dose and schedule for phase II trials was $1500 \mu \mathrm{g} / \mathrm{m}^{2}$ as a 24-hour continuous infusion repeated every 3 weeks (Garcia-Carbonero et al 2004; Yovine et al 2004; Le Cesne et al 2005). The most commonly observed toxicities in phase II trials were neutropenia, thrombocytopenia and elevation of liver enzymes (Table 1).

\section{Prevention of severe toxicity}

Pharmacokinetic studies conducted during the phase I trials have allowed the identification of several factors predictive of severe toxicity during treatment with trabectedin (Gomez et al 2000; Taamma et al 2001). Most notably abnormal serum alkaline phosphatase level at baseline was a predictor of liver enzymes elevation (Taamma et al 2001) and baseline serum bilirubin above $0.6 \times \mathrm{ULN}$ was a predictor of severe toxicity (haematological and hepatic).

Grosso, Dileo et al (2006) recently reported the use of steroid premedication to prevent biochemical hepatic and haematological toxicity. In this retrospective study, 54 patients with STS, osteosarcoma or Ewing tumors received trabectedin in a compassionate-use program between April 2000 and 2005. Based on the observation that pre-treatment with dexamethasone significantly reduced hepatotoxicity of trabectedin in rats (Donald et al 2003), patients treated after April 2002 received dexamethasone $4 \mathrm{mg}$ twice daily beginning the day before administration of trabectedin. Twenty-three patients were treated without dexamethasone premedication and 31 received dexamethasone prior to trabectedin. In the group receiving dexamethasone the

Table I Main toxicities observed in phase II trials in patients with soft tissue sarcoma

\begin{tabular}{|c|c|c|c|c|c|c|}
\hline Reference & Study type & $\mathbf{N}$ & $\begin{array}{l}\text { Grade } 3-4 \\
\text { neutropenia }\end{array}$ & $\begin{array}{l}\text { Febrile } \\
\text { neutropenia }\end{array}$ & $\begin{array}{l}\text { Grade 3-4 } \\
\text { thrombocytopenia }\end{array}$ & $\begin{array}{l}\text { Grade } 3-4 \\
\text { transaminitis }\end{array}$ \\
\hline Delaloge et al 200I & $\begin{array}{l}\text { Phase I + compassionate- } \\
\text { use program }\end{array}$ & 29 & $48.4 \%$ & $10.3 \%$ & $20.6 \%$ & $52.0 \%$ \\
\hline Yovine et al 2004 & Phase II, second line & 54 & $61 \%$ & $7 \%$ & $19 \%$ & $\begin{array}{l}48 \% \text { ALT } \\
57 \% \text { ALT }\end{array}$ \\
\hline $\begin{array}{l}\text { Garcia-Carbonero } \\
\text { et al } 2004\end{array}$ & Phase II, second line & 36 & $34 \%$ & $6 \%$ & $17 \%$ & $\begin{array}{l}26 \% \text { AST } \\
20 \% \text { ALT }\end{array}$ \\
\hline Le Cesne et al 2005 & Phase II, second line & 104 & $52.5 \%$ & $9.1 \%$ & $18.2 \%$ & $\begin{array}{l}35.3 \% \text { AST } \\
44.5 \% \text { ALT }\end{array}$ \\
\hline $\begin{array}{l}\text { Garcia-Carbonero } \\
\text { et al } 2005\end{array}$ & Phase II, first line & 36 & $33 \%$ & $0 \%$ & $0 \%$ & $\begin{array}{l}34 \% \text { AST } \\
36 \% \text { ALT }\end{array}$ \\
\hline Huygh et al 2006 & $\begin{array}{l}\text { Phase II + compassionate- } \\
\text { use program }\end{array}$ & 89 & $35.9 \%$ & $7.9 \%$ & $17.9 \%$ & $34.9 \%$ \\
\hline
\end{tabular}


incidence of grade 3-4 liver enzyme elevation, neutropenia and thrombocytopenia fell to $3 \%, 10 \%$, and $0 \%$ respectively, compared with $70 \%, 39 \%$, and $35 \%$ respectively in the group without premedication ( $\mathrm{p}<0.0001$ using the $\chi^{2}$ for elevated liver enzymes and neutropenia, no p-value reported for the difference in the rate of thrombocytopenia). The progression free survival (PFS) was comparable in both groups (no numerical nor p-values reported) and the PFS for the whole cohort was in the range of that reported in phase II trials. Since these data are derived from a retrospective study they need to be validated in a prospective trial before steroid premedication can be safely used in clinical practice. Indeed, although steroid premedication seems to lower trabectedin toxicity, the mechanism by which it does so in humans is still unknown, and one may wonder whether or not dexamethasone influences the pharmacokinetics of trabectedin. In the rat model described by Donald and colleagues dexamethasone premedication lowered the levels of trabectedin in the liver (both maximal concentration and area under the curve) without lowering the plasma level (Donald et al 2003), however these data need to be confirmed in humans.

\section{Efficacy of trabectedin in sarcoma Trabectedin in non-gastrointestinal stromal tumors (non-GIST) STS}

The first paper to specifically report clinical activity of trabectedin in STS was published in 2001 by Delaloge et al (2001). This study included 12 patients with STS entered in the phase I reported by Taamma et al (2001) and 17 patients with STS entered in a compassionate-use program. Overall the author observed 4 partial responses (PR) (14\%), 10 disease stabilisation (SD) (34\%) lasting more than 2 months. The median time to progression was 2.8 months. Eight patients $(28 \%)$ were progression free at 6 months and 4 (14\%) were progression-free at 12 months. These results were quite impressive considering that all patients had received both doxorubicin and an alkylating agent prior to treatment with trabectedin.

Yovine et al (2004) reported the results a French phase II trial of trabectedin $1500 \mu \mathrm{g} / \mathrm{m}^{2}$ administered as a 24-hour continuous infusion in patients with progressive STS after at least one prior chemotherapy regimen. Fifty-four patients were accrued between February 1999 and January 2001 into 2 different cohort based on prior treatment: group 1, 1 or 2 drugs $(n=26)$; group 2, 3 or more drugs $(n=28)$. All patients in this trial had a good ECOG performance status (PS) (0 or 1). Leiomyosarcoma was the predominant histological subtype $(n=22,41 \%$, with uterine origin in 8 of
22 cases) followed by liposarcoma (11\%), 4 patients (7\%) had GIST. All patients had metastatic disease at the time of study entry. Two patients had PR (3.7\%), 9 patients (17\%) had SD for more than 6 months and 28 patients $(51.9 \%)$ had progressive disease. After a median follow-up of 26 months, the median PFS was 1.9 months, and $38.8 \%$ and $24.1 \%$ of patients were progression-free at 3 and 6 months respectively. Of note the PFS and the 3 and 6 months progression-free rate were similar between the two groups. Median overall survival was 12.8 months, and was longer in group 1 than in group 2 (14 versus 8 months respectively) (Yovine et al 2004).

Garcia-Carbonero et al reported the results of the American phase II trial of trabectedin $1500 \mu \mathrm{g} / \mathrm{m}^{2}$ administered as a 24-hour continuous infusion in 36 patients with progressive STS after failure of at least one prior chemotherapy regimen (Garcia-Carbonero et al 2004). This study enrolled 36 patients between September 1999 and September 2000. All patients had metastatic disease, good ECOG PS $(0-1)$ and most of them had either leiomyosarcoma $(n=13$, $36 \%)$ or liposarcoma $(n=10,28 \%)$. All patients were also previously treated with anthracycline containing regimens. One complete response (CR), 2 PR (objective response rate, $\mathrm{CR}+\mathrm{PR}=8 \%$ ) were observed. Median PFS was 1.7 months and the 6 and 12 months progression-free rate were approximately $20 \%$ and $10 \%$. Median overall survival was 12.1 months

A third study of trabectedin $1500 \mu \mathrm{g} / \mathrm{m}^{2}$ as a 24-hour continuous infusion was conducted by the EORTC and reported by Le Cesne et al (2005). This trial accrued 104 patients with non-GIST STS failing prior chemotherapy, as well as 28 patients with progressive GIST (Blay et al 2004; Le Cesne et al 2005). The results of trabectedin in patients with GIST were reported separately and will be discussed in the next paragraph. All patients treated in the STS cohort had good PS (0-1), the most common histological subtypes were leiomyosarcoma (41\%), synovial sarcoma (17\%) and liposarcoma (10\%). There were 8 (7.7\%) PR and 45 (43.3\%) SD. After a median follow-up of 34 months, the median PFS was 3.4 months (105 days) and the median overall survival was 9.2 months. Fifty-two percent, $29 \%$ and $17 \%$ of patients were progression-free at 3, 6 and 12 months respectively.

A single institution's experience was reported by investigators from the Leuven Cancer Institute in Leuven, Belgium (Huygh et al 2006). This report addresses the outcome of 89 patients, 15 of whom were treated in a phase II trial, while 74 other patients were treated on a compassionate-use program. Most patients had leiomyosarcoma (33\%), liposarcoma 
(18\%) or osteosarcoma (16\%), and all but 3 had received prior chemotherapy, in most cases with doxorubicin (93\%). The objective response rate in this heterogeneous cohort was 7\% (1 CR and 5 PR), and 32 disease stabilization (36\%) were also observed. Median PFS and overall survival were 2.0 and 8.2 months respectively. Toxicity in this unselected population was similar to that observed in phase II trials (Table 1).

Based on the interesting results seen in patients failing prior chemotherapy, a phase II trial in chemotherapy-naive patients with advanced STS was launched in September 1999 (Garcia-Carbonero et al 2005). This study accrued 36 patients with good ECOG PS (0-1), most of whom had either leiomyosarcoma (42\%) or liposarcoma (25\%). Six patients had PR (17\%) for a median duration of 16.5 (range 5.1-32.5) months. Overall the median PFS was 1.6 months and the median overall survival was 15.8 months, however the 6- and 12-months PFS rates were 24.4 and $21 \%$ indicating that approximately $20 \%$ of patients achieve long-term tumor control. The main efficacy outcomes of trabectedin in these phase II trials are reported in Table 2.

A randomized phase II trial comparing trabectedin given at $580 \mu \mathrm{g} / \mathrm{m}^{2}$ on a weekly schedule to the "standard" $1500 \mu \mathrm{g} / \mathrm{m}^{2}$ 24-hour continuous infusion every 3 weeks schedule in patients with leiomyosarcoma or liposarcoma failing prior therapy with doxorubicin and ifosfamide was first reported at the 2004 ASCO meeting (Samuels et al 2004) was updated at the 2007 ASCO meeting (Morgan et al 2007). Two hundred and seventy patients were accrued, all had received at least one prior chemotherapy regimen (median 2, range 1-6). Progression-free survival was statistically superior in the standard, $1500 \mu \mathrm{g} / \mathrm{m}^{2}$ 24-hour continuous infusion every 3 weeks, arm (3.3 vs 2.3 months, $p=0.0418$ ), while OS was not statistically different between arms (13.8 vs 11.8 months, $\mathrm{p}=0.1984$ ). More neutropenia, increase in liver enzymes, emesis and fatigue occurred in the standard dosing arm (Morgan et al 2007).

\section{Trabectedin in patients with GIST}

Two trials have investigated the efficacy of trabectedin in GISTs. In both of these trials trabectedin was found to be inefficient in this disease. The first trial was reported by Ryan et al (2002) and included 20 patients, most of whom were chemotherapy-naïve (55\%). Trabectedin was administered as a 24 -hour continuous infusion of $1500 \mu \mathrm{g} / \mathrm{m}^{2}$ every 3 weeks. The best response in this study was stable disease, which was observed in 2 patients (10\%), lasting 4 and 10 cycles (12 and 30 weeks). The median PFS was 1.25 months, and the overall survival was not reached after more than 15 months of median follow-up. However this extended overall survival was due to the fact that most patients received imatinib (GLIVEC ${ }^{\circledR}$ ) GLEEVEC $^{\circledR}$, Novartis, Basel, Switzerland) after failure of trabectedin. The median survival of the 7 patients who did not receive imatinib was 8.6 months (Ryan et al 2002).

Another trial, led by the EORTC and reported by Blay et al (2004), enrolled 28 patients, trabectedin was given as a 24-hour continuous infusion of $1500 \mu \mathrm{g} / \mathrm{m}^{2}$ every 3 weeks. Patients were required by protocol to be chemotherapy-naïve. No objective responses were seen but 9 patients (33\%) had stable disease at the first response assessment ( 2 cycles). The median PFS was 51 days (1.7 months) and the median overall survival was 589 days (19.3 months), here again reflecting the fact that the 18 patients who received "further medical treatment after trabectedin" likely received imatinib (Blay et al 2004).

\section{Factors predicting response to trabectedin}

All phase II trials have shown that trabectedin, despite a relatively low response rate, could induce long term tumor

Table 2 Main efficacy outcomes of trabectedin in phase II trials in patients with non-GIST soft tissue sarcoma

\begin{tabular}{|c|c|c|c|c|c|c|}
\hline Reference & Study type & $\mathbf{N}$ & $\begin{array}{l}\text { Response } \\
\text { rate }\end{array}$ & $\begin{array}{l}\text { Median PFS } \\
\text { (months) }\end{array}$ & $\begin{array}{l}\text { 6-month } \\
\text { PFS }\end{array}$ & $\begin{array}{l}\text { Median OS } \\
\text { (months) }\end{array}$ \\
\hline Delaloge et al 200I & $\begin{array}{l}\text { phase I + compassionate- } \\
\text { use program }\end{array}$ & 29 & $14.0 \%$ & 2.8 & $28.0 \%$ & NR \\
\hline Yovine et al 2004 & Phase II, second line & 54 & $3.7 \%$ & 1.9 & $24.1 \%$ & 12.8 \\
\hline $\begin{array}{l}\text { Garcia-Carbonero } \\
\text { et al } 2004\end{array}$ & Phase II, second line & 36 & $8.0 \%$ & 1.7 & $20.0 \%$ & 12.1 \\
\hline Le Cesne et al 2005 & Phase II, second line & 104 & $8.0 \%$ & 3.4 & $29.0 \%$ & 9.2 \\
\hline $\begin{array}{l}\text { Garcia-Carbonero } \\
\text { et al } 2005\end{array}$ & Phase II, first line & 36 & $17.1 \%$ & 1.6 & 24.4 & 15.8 \\
\hline Huygh et al 2006 & $\begin{array}{l}\text { Phase II + compassionate- } \\
\text { use program }\end{array}$ & 89 & $7.0 \%$ & 2.0 & NR & 8.2 \\
\hline
\end{tabular}

NR, not reported. 
control in approximately $15-20 \%$ of patients, several retrospective studies have focused on the identification of these patients.

Grosso et al (Grosso, Demetri et al 2006a; Grosso, Jones et al 2007) reported on the activity of trabectedin in myxoid liposarcoma. Myxoid liposarcoma contains a characteristic chromosomal translocation, $\mathrm{t}(12 ; 16)$, resulting in the fusion of a transcription factors (CHOP) and an RNA binding protein (TLS/FUS) (Crozat et al 1993). At least 3 different fusion products have been identified, based on the TLS/FUS breakpoint, but were not shown to have prognostic significance (Antonescu et al 2001). In the retrospective analysis performed by Grosso, Forni et al (2007), 51 patients with myxoid liposarcoma were treated in 5 different European and American institutions. The overall response rate using RECIST criteria was 51\% $(C R=4 \%$, PR $=47 \%)$, with SD seen in $39 \%$ patients. The 6 months PFS rate was $88 \%$ and the median PFS was 14 months (Grosso, Jones et al 2007). Preliminary data based on the observation made on 12 patients for whom fresh frozen tissue was available for RT-PCR experiments showed that probability of response was influenced by the type of fusion transcript present in the tumor: 2 patients with type III fusion transcript had disease progression as their best response (PFS 1.3 months in both patients), while the remaining 10 patients who had type I, II and/or IV fusion transcript had at least a PR following treatment with trabectedin (PFS ranging from 6.7 to 20.5 months) (Grosso, Jones et al 2007). In an in vitro study using primary cell culture, Grosso et al also reported that in the myxoid liposarcoma cell line carrying the type I transcript, impairment of the selective binding of FUS-CHOP to some promoters was observed after treatment with trabectedin at concentration 1-4 nM (Grosso, Forni et al 2007).

Based on the observation that functional status of both NER and HRR influences the activity of trabectedin in preclinical models (Herrero et al 2006), Schöffski et al (2006) conducted a retrospective analysis of the NER and HRR status, using RT-PCR, on the tumors of 92 patients treated with trabectedin. In this study patients with higher ERCC1 expression (part of the NER machinery) had improved 6-month PFS rate and median overall survival compared with patients whose tumor expressed lower levels of ERCC1 (32 versus $15 \%, \mathrm{p}=0.07$ and 12 versus 7 months respectively). Furthermore patients whose tumor expressed lower levels of BRCA1 (part of the HRR system) also had improved 6-month PFS rate and median overall survival compared with patients whose tumor expressed high levels of BRCA1 (33 vs $11 \%, p=0.02$ and 15 vs 5 months, $\mathrm{p}=0.0003$, for
PFS and OS respectively). BRCA2 (HRR system) and XPD (NER system) expressions had no significant impact on PFS and survival. A combined analysis of ERCC1 and BRCA1 expression identified a subgroup of patients with high ERCC1 and low BRCA1 expression who had a 50\% 6-month PFS rate $(\mathrm{p}=0.0003)$ and a 20.4 months median overall survival $(\mathrm{p}=0.0005)$. Multivariate analysis showed that both ERCC1 (hazard ratio for progression for high level $=0.52, p=0.02$ ) and BRCA1 (hazard ratio for progression for high expression level $=2.73, \mathrm{p}=0.0006$ ) expression levels were independent predictors of PFS and that only BRCA1 independently predicted overall survival (hazard ratio for death for high level $=2.57, \mathrm{p}=0.0005$ ).

\section{Perspective and future developments}

Several trabectedin-based combinations have been tested in phase I trials, including combination with cisplatin (Grasselli et al 2007) capecitabine (Gore et al 2006), paclitaxel (Papadopoulos et al 2006), docetaxel (Von Mehren et al 2006) and pegylated doxorubicin (Cohen et al 2005). These trials have shown that combination therapy with trabectedin was feasible and induced responses or prolonged disease stabilizations in tumor types known to be sensitive either to trabectedin or to the drug it was combined to. Neither major drug-drug interactions nor major unpredicted toxicity were observed in these trials. Several of these combination are based on the observation of synergy in preclinical models: cisplatin-trabectedin combination for example was shown to be synergistic in various human tumor xenografts (D'Incalci et al 2003) and treatment of sarcoma or breast cancer cells with doxorubicin or paclitaxel 24 hours prior to treatment with trabectedin was shown to be synergistic, both in vitro and in vivo in nude mice (Takahashi et al 2001, 2002).

Another direction of ongoing research is the identification of patients with a high probability of benefit from treatment with trabectedin. Preliminary data reported at the 2006 and 2007 ASCO meetings regarding predictive factors in patients undergoing treatment with trabectedin (Grosso, Demetri et al 2006; Schoffski et al 2006; Grosso, Forni et al 2007; Grosso, Jones et al 2007) will need validation in larger cohorts and possibly in prospective trials before treatment can be allocated based on these factors.

\section{Conclusion}

Trabectedin binds the DNA minor groove and has an original mechanism of action involving proteins of the nucleotide excision repair machinery. Trabectedin has shown promising 
activity in patients with STS, with long-term (12 months) disease control achieved in approximately $20 \%$ of patients in first line advanced disease and approximately $15 \%$ in second line chemotherapy. In patients with normal hepatic function assessed by normal bilirubin and alkaline phosphatases (ALP), trabectedin can be safely administered at $1500 \mu \mathrm{g} / \mathrm{m}^{2}$ every 3 weeks, the dose should however be lowered to $1200 \mu \mathrm{g} / \mathrm{m}^{2}$ in case of a rise in bilirubin or ALP between cycles (Le Cesne et al 2005). Development in patients with other malignancies such as breast and ovarian carcinoma is currently ongoing. Further development in STS should be based on biological and/or histological factors predictive of response.

\section{Abbreviations}

ASCO, American Society of Clinical Oncology; DLT, dose-limiting toxicity; ECOG, Eastern Cooperative Oncology Group, GIST, gastro-intestinal stromal tumor; HRR, homologous recombination repair: MTD, maximum tolerated dose; NER, nucleotide-excision repair; OS, overall survival; PD, progressive disease; PFS, progression-free survival; PR, partial response; PS, performance status; $\mathrm{SD}$, stable disease; STS, soft tissue sarcoma.

\section{References}

Antman K, Crowley J, Balcerzak SP, et al. 1993. An intergroup phase III randomized study of doxorubicin and dacarbazine with or without ifosfamide and mesna in advanced soft tissue and bone sarcomas. J Clin Oncol, 11:1276-85.

Antonescu CR, Tschernyavsky SJ, Decuseara R, et al. 2001. Prognostic impact of P53 status, TLS-CHOP fusion transcript structure, and histological grade in myxoid liposarcoma: a molecular and clinicopathologic study of 82 cases. Clin Cancer Res, 7:3977-87.

Bay JO, Ray-Coquard I, Fayette J, et al. 2006. Docetaxel and gemcitabine combination in 133 advanced soft-tissue sarcomas: a retrospective analysis. Int J Cancer, 119:706-11.

Blay JY, Le CA, Verweij J, Scurr M, et al. (2004). A phase II study of ET743/trabectedin ('Yondelis') for patients with advanced gastrointestinal stromal tumours. Eur J Cancer, 40:1327-31.

Clark MA, Fisher C, Judson I, et al. 2005. Soft-tissue sarcomas in adults. N Engl J Med, 353:701-11.

Cohen RB, Schilder RJ, Cheng J, et al. 2005. Final results of a combination study between trabectedin and pegylated. ASCO Meeting Abstracts, 23:3074.

Crozat A, Aman P, Mandahl N, et al. 1993. Fusion of CHOP to a novel RNAbinding protein in human myxoid liposarcoma. Nature, 363:640-4.

D'Incalci M, Colombo T, Ubezio P, et al. 2003. The combination of yondelis and cisplatin is synergistic against human tumor xenografts. Eur J Cancer, 39:1920-6.

David-Cordonnier MH, Gajate C, Olmea O, et al. 2005. DNA and non-DNA targets in the mechanism of action of the antitumor drug trabectedin. Chem Biol, 12:1201-10.

Delaloge S, Yovine A, Taamma A, et al. 2001. Ecteinascidin-743: a marinederived compound in advanced, pretreated sarcoma patients - preliminary evidence of activity. J Clin Oncol, 19:1248-55.

Donald S, Verschoyle RD, Greaves P, et al. 2003. Complete protection by high-dose dexamethasone against the hepatotoxicity of the novel antitumor drug yondelis (ET-743) in the rat. Cancer Res, 63:5902-8.
Edmonson JH, Ryan LM, Blum RH, et al. 1993. Randomized comparison of doxorubicin alone versus ifosfamide plus doxorubicin or mitomycin, doxorubicin, and cisplatin against advanced soft tissue sarcomas. JClin Oncol, 11:1269-75.

Friedman D, Hu Z, Kolb EA, et al. 2002. Ecteinascidin-743 inhibits activated but not constitutive transcription. Cancer Res, 62:3377-81.

Garcia-Carbonero R, Supko JG, Maki RG, et al. 2005. Ecteinascidin-743 (ET-743) for chemotherapy-naive patients with advanced soft tissue sarcomas: multicenter phase II and pharmacokinetic study. J Clin Oncol, 23:5484-92.

Garcia-Carbonero R, Supko JG, Manola J, et al. 2004. Phase II and pharmacokinetic study of ecteinascidin 743 in patients with progressive sarcomas of soft tissues refractory to chemotherapy. J Clin Oncol, 22:1480-90.

Gomez J, Lopez-Lazaro L, Guzman C, et al. 2000. Identification of biochemical parameters that predict the onset of severe toxicities in patients treated with ET-743. ASCO Meeting Abstracts, 19:727.

Gore L, Rivera E, Lavallee K, et al. 2006. Phase I combination study of trabectedin (T) and capecitabine (C) in patients with advanced malignancies. ASCO Meeting Abstracts, 24:2079.

Grasselli G, Malossi A, Colombo N, et al. 2007. Phase I and pharmacokinetic (PK) study of ecteinascidin-743 (ET, Trabectedin) and cisplatin (P) combination in pre-treated patients (pts) with selected advanced solid tumors. ASCO Meeting Abstracts, 22:542.

Grosso F, Demetri GD, Blay JY, et al. 2006. Patterns of tumor response to trabectedin (ET743) in myxoid liposarcomas. ASCO Meeting Abstracts, 24:9511.

Grosso F, Dileo P, Sanfilippo R, et al. 2006. Steroid premedication markedly reduces liver and bone marrow toxicity of trabectedin in advanced sarcoma. Eur J Cancer, 42:1484-90.

Grosso F, Forni C, Frapolli R, et al. 2007). Sensitivity of myxoid-round cell liposarcoma (MRCL) to trabectedin (T) may be related to a direct effect on the fusion transcript. ASCO Meeting Abstracts, 25:10000.

Grosso F, Jones RL, Demetri GD, et al. 2007. Efficacy of trabectedin (ecteinascidin-743) in advanced pretreated myxoid liposarcomas: a retrospective study. Lancet Oncol, 8:595-602.

Hensley ML, Maki R, Venkatraman E, et al. 2002. Gemcitabine and docetaxel in patients with unresectable leiomyosarcoma: results of a phase II trial. J Clin Oncol, 20:2824-31.

Herrero AB, Martin-Castellanos C, Marco E, et al. 2006. Cross-talk between nucleotide excision and homologous recombination DNA repair pathways in the mechanism of action of antitumor trabectedin. Cancer Res, 66:8155-62.

Huygh G, Clement PM, Dumez H, et al. 2006. Ecteinascidin-743: evidence of activity in advanced, pretreated soft tissue and bone sarcoma patients. Sarcoma, 2006:56282.

, Blay JY, Judson I, et al. 2005. Phase II study of ET-743 in advanced soft tissue sarcomas: a European Organisation for the Research and Treatment of Cancer (EORTC) soft tissue and bone sarcoma group trial. J Clin Oncol, 23:576-84.

Morgan JA, Le Cesne A, Chawla S, et al. 2007. Randomized phase II study of trabectedin in patients with liposarcoma and leiomyosarcoma (L-sarcomas) after failure of prior anthracylines (A) and ifosfamide (I). ASCO Meeting Abstracts, 25:10060.

Papadopoulos KP, Chu Q, Patnaik A, et al. 2006. Phase I and pharmacokinetics (PK) study of sequential paclitaxel and trabectedin every 2 weeks in patients with advanced solid tumors. ASCO Meeting Abstracts, 24:2029.

Patel SR, Gandhi V, Jenkins J, et al. 2001. Phase II clinical investigation of gemcitabine in advanced soft tissue sarcomas and window evaluation of dose rate on gemcitabine triphosphate accumulation.. J Clin Oncol, 19:3483-9.

Ryan DP, Puchalski T, Supko JG, et al. 2002. A phase II and pharmacokinetic study of ecteinascidin 743 in patients with gastrointestinal stromal tumors. Oncologist, 7:531-8.

Ryan DP, Supko JG, Eder JP, et al. 2001. Phase I and pharmacokinetic study of ecteinascidin 743 administered as a 72 -hour continuous intravenous infusion in patients with solid malignancies. Clin Cancer Res, 7:231-42. 
Samuels BL, Rushing D, Chawla SP, et al. 2004. Randomized phase II study of trabectedin (ET-743) given by two. ASCO Meeting Abstracts, 22:9000.

Schoffski P, Casali PG, Taron M, et al. 2006. DNA repair functionality modulates the clinical outcome of patients. ASCO Meeting Abstracts, 24:9522.

Soares DG, Escargueil AE, Poindessous V, et al. 2007. From the cover: replication and homologous recombination repair regulate DNA doublestrand break formation by the antitumor alkylator ecteinascidin 743 . Proc Natl Acad Sci USA, 104:13062-7.

Taamma A, Misset JL, Riofrio M, et al. 2001. Phase I and pharmacokinetic study of ecteinascidin-743, a new marine compound, administered as a 24-hour continuous infusion in patients with solid tumors. $J$ Clin Oncol, 19:1256-65.

Takahashi N, Li W, Banerjee D, et al. 2002. Sequence-dependent synergistic cytotoxicity of ecteinascidin-743 and paclitaxel in human breast cancer cell lines in vitro and in vivo. Cancer Res, 62:6909-15.

Takahashi N, Li WW, Banerjee D, et al. 2001. Sequence-dependent enhancement of cytotoxicity produced by ecteinascidin 743 (ET-743) with doxorubicin or paclitaxel in soft tissue sarcoma cells. Clin Cancer Res, 7:3251-7.

Takebayashi Y, Goldwasser F, Urasaki Y, et al. 2001. Ecteinascidin 743 induces protein-linked DNA breaks in human colon carcinoma HCT116 cells and is cytotoxic independently of topoisomerase I expression. Clin Cancer Res, 7:185-91.
Takebayashi Y, Pourquier P, Zimonjic DB, et al. 2001. Antiproliferative activity of ecteinascidin 743 is dependent upon transcription-coupled nucleotide-excision repair. Nat Med, 7:961-66.

Twelves C, Hoekman K, Bowman A, et al. 2003. Phase I and pharmacokinetic study of Yondelis (Ecteinascidin-743; ET-743) administered as an infusion over $1 \mathrm{~h}$ or $3 \mathrm{~h}$ every 21 days in patients with solid tumours. Eur J Cancer, 39:1842-51.

Villalona-Calero MA, Eckhardt SG, Weiss G, et al. 2002. A phase I and pharmacokinetic study of ecteinascidin-743 on a daily $\times 5$ schedule in patients with solid malignancies. Clin Cancer Res, 8:75-85.

Von Mehren M, Buck D, Temmer E, et al. 2006. Phase I study of trabectedin (T) in combination with docetaxel (D) in patients with advanced malignancies. ASCO Meeting Abstracts, 24:2068.

Wilson RE, Wood WC, Lerner HL, et al. 1986. Doxorubicin chemotherapy in the treatment of soft-tissue sarcoma. Combined results of two randomized trials. Arch Surg, 121:1354-9.

Yovine A, Riofrio M, Blay JY, et al. 2004. Phase II study of ecteinascidin743 in advanced pretreated soft tissue sarcoma patients. J Clin Oncol, 22:890-9. 